\title{
Standard Definitions of Terms Relating to Mass Spectrometry
}

\section{A Report from the Committee on Measurements and Standards of the American Society for Mass Spectrometry}

\author{
Phil Price* \\ Union Carbide Technical Center, South Charleston, West Virginia, USA
}

(J Am Soc Mass Spectrom 1991, 2, 336-348)

$\mathrm{T}$ The following document is the latest revision of Standard Definitions of Terms Relating to Mass Spectrometry. The present version was begun by merging the last ASMS "Committee on Nomencla-

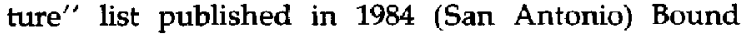
Volume with the "IUPAC Working Document on Mass Spectrometry Nomenclature,' from 1987 and 1985. Duplications were removed, terms added, and a few updated. This compilation was circulated for editorial comment at the 1987 ASMS Conference in Denver, and reviewed further at the 1988 meeting in San Francisco. During 1988-1989, as a result of a Spectaker article, about 70 ASMS members requested (and were supplied) copies of the draft definitions. A few comments were received, and most were incorporated into the document. During 1989-1990, Rodger Foltz, Chairman of the ASMS Measurements and Standards Committee, circulated copies to a select group of spectroscopists and requested that each review a specific section. Their additional comments were incorporated into the present document. Over 100 members of ASMS have assisted with these definitions by reading, editing, and supplying new defunitions. Particularly detailed and extensive suggestions from Bill Budde, Ken Busch, Doug Cameron, Hank Fales, Rodger Foltz, Keith Jennings, Richard Martinez, John Todd, and J. Throck Watson were very helpful and are appreciated.

These definitions are closely parallel to IUPAC, and efforts have been made to avoid conflicts with IUPAC terminology. This compilation is presented as a working document, with the intention to update the recommended terminology list regularly. There is now an organized effort to collect new terms, accept revisions to existing terms, and provide a node for discussions of conflicting and potentially misleading or confusing terminology. Care must be taken not to

\footnotetext{
* This report was compiled by Dr. Price for the American society for Mass Spectrometry.

Address reprint requests to Phil Price, Building 770-120, Union Carbide Technical Center, Kanawha Turnpike, South Charleston, WV 25303.
}

arbitrarily add terminology before adequate discussion and agreement among ASMS members. Often, this discussion process will prevent "timely addition's of new terms, but will avoid addition of terms only accepted by a small part of the community. The goal is to provide an educational tool, and editorial guide, and a generally accepted set of definitions to foster clearer communications among mass spectrometrists (and with "outsiders").

Many terms have been omitted from the list. This will only be remedied by participation and discussion among the membership. All comments regarding either this set of terms or potential additions are welcome!

Contact: Chair, Committee on Measurements and Standards

c/o ASMS Office

P.O. Box 1508

East Lansing, MI 48826

Phone: (517) 337-2548

Fax: (517) 332-7503

or

Phil Price

Building 770-120

Union Carbide Technical Center

Kanawha Turnpike

South Charleston, WV 25303

Phone: (304) 747-5594

Fax: (304) 747-5430

\section{Standard Definitions of Terms Relating to} Mass Spectrometry

\begin{tabular}{lc} 
Topic & Page number \\
\hline Analyzers & $\mathbf{3 3 7}$ \\
Data system & $\mathbf{3 3 8}$ \\
Sample introduction & $\mathbf{3 3 9}$ \\
Scanning of spectra & $\mathbf{3 4 0}$ \\
lon detection and sensitivity & $\mathbf{3 4 2}$ \\
lonization nomenclature & 343 \\
Types af ions, ion structures & $\mathbf{3 4 5}$ \\
lon/molecule reactions & $\mathbf{3 4 7}$ \\
Vacuum & $\mathbf{3 4 8}$
\end{tabular}




\section{Analyzers}

Mass spectrometer. An instrument in which ions are analyzed according to their mass-to-charge ratio, and in which the number of ions is determined electrically (or scintillator, vidicon, etc.).

Mass spectrograph. An instrument in which beams of ions are separated according to their mass-to-charge ratio, and in which the deflection and intensity of the beams are recorded directly on a photographic plate or film.

Single-focusing mass spectrometer. An instrument in which ions with a given mass-to-charge ratio are brought to a focus although the initial directions of the ions diverge.

Nier-Johnson geometry. An arrangement for a double-focusing mass spectrometer in which a deflection of $\pi / 2$ radians in a radial electrostatic field analyzer is followed by a magnetic deflection of $\pi / 3$ radians. The electrostatic analyzer uses a symmetrical object-image arrangement and the magnetic analyzer is used asymmetrically.

Mattauch-Herzog geometry. An arrangement for a double-focusing mass spectrometer in which a deflection of $\pi / 4 \sqrt{ } 2$ radians in a radial electrostatic field is followed by a magnetic deflection of $\pi / 2$ radians.

Static fields mass spectrometer. A mass spectrometer that can separate ion beams with fields that do not vary with time. The fields are generally electrostatic and magnetic.

Dynamic field(s) mass spectrometer. A mass spectrometer in which the separation of an ion beam depends essentially on the use of a field, or fields that very with time. These fields are generally electrostatic and magnetic.

$m / z$. An abbreviation used to denote the dimensionless quantity formed by dividing the mass of an ion by the number of charges carried by the ion. It has long been called the mass-to-charge ratio although $m$ is not the ionic mass nor is $z$ a multiple of the electronic charge, $\mathrm{e}^{-}$. The abbreviation $m / e$, therefore, is not recommended. Thus, for example, for the ion $\mathrm{C}_{7} \mathrm{H}_{7}^{2+}, m / z=45.5$.

Nominal ion mass. The mass of an ion with a given empirical formula calculated using the integer mass numbers of the most abundant isotope of each element, e.g., $\mathrm{C}=12, \mathrm{H}=1, \mathrm{O}=16$.

Monoisotopic ion mass. The mass of an ion for a given empirical formula calculated using the exact mass of the most abundant isotope of each element, e.g., $\mathrm{C}=12.000000, \mathrm{H}=1.0017825, \mathrm{O}=15.994915$.
Average mass. The mass of an ion for a given empirical formula calculated using the atomic weight of each element, e.g., $\mathrm{C}=12.01115, \mathrm{H}=1.00797, \mathrm{O}$ $=15.9994$.

Electrostatic analyzer. A velocity-focusing device for producing an electrostatic field perpendicular to the direction of ion travel (usually used in combination with a magnetic analyzer for mass analysis). The effect is to bring to a common focus all ions of a given kinetic energy.

Magnetic analyzer. A direction-focusing device that produces a magnetic field perpendicular to the direction of ion travel. The effect is to bring to a common focus all ions of a given momentum with the same mass-to-charge ratio.

Quadrupole analyzer. A mass filter that creates a quadrupole field with a dc component and an if component in such a manner as to allow transmission only of ions having a selected mass-to-charge ratio.

Time-of-fight analyzer. A device that measures the flight time of ions with an equivalent kinetic energy over a fixed distance.

Wien analyzer. A velocity filter with crossed homogeneous electric and magnetic fields, for transmitting only ions of a fixed velocity.

Mass resonant analyzer. A mass analyzer for massdependent resonant energy transfer and measurement of the resonance frequency, power, or ion current of the resonant ions.

Double-focusing analyzer. A magnetic analyzer and an electrostatic analyzer combined in either sequence to effect direction and velocity focusing.

Ion cyclotron resonance analyzer. A device to determine the mass-to-charge ratio of an ion in the presence of a magnetic field by measuring its cyclotron frequency.

Ion trap analyzer. A mass-resonance analyzer that produces a three-dimensional rotationally symmetric quadrupole field capable of storing ions at selected mass-to-charge ratios.

Mass spectrometer configuration. Multianalyzer instruments should be named for the analyzers in the sequence in which they are traversed by the ion beam, where B is for a magnetic analyzer, $E$ for an electrostatic analyzer, $Q$ for a quadrupole analyzer, TOF for time-of-flight analyzer, and ICR for an ion cyclotron resonance analyzer. For example, a BE mass spectrometer ("reversed" geometry double-focusing instrument), BQ mass spectrometer (hybrid sector and 
quadrupole instrument), EBQ (double-focusing instrument followed by a quadrupole).

Mass analysis. A process by which a mixture of ionic (or neutral) species is separated according to the mass-to-charge $(\mathrm{m} / \mathrm{z}$ ) ratios (for ions) or their aggregate atomic masses (for neutrals). The analysis may be qualitative and/or quantitative.

Vacuum system. Components associated with lowering the pressure within a mass spectrometer. A vacuum system includes not only the various pumping components but also valves, gauges, and associated electronic or other control devices, the chamber in which ions are formed and detected, and the vacuum envelope.

\section{Data System}

Data system. Components used to record and process information during the analysis of a sample. The system includes a computer and an analog-to-digital conversion module, as well as other control devices for data recording, storage, and manipulation.

Data processing. Once information is obtained with an appropriate data system, the information must be interpreted appropriately for the end use. Those steps that lead to this end use are of concern in data processing. Data processing does not necessarily imply application of modern computer techniques.

Data reduction. The process of transforming the initial digital or analog representation of output from a spectrometer into a form that is amenable to interpretation, e.g., a bar graph, a table of masses versus intensities.

Data acquisition. The process of transforming spectrometer signals from their original form into suitable representations, with or without modification, with or without a computer system.

Real time. A data acquisition method in which the mass spectra are generated within the same time frame as the original experiment.

Off-line. A data acquisition method in which the mass spectra are produced some time after the original experiment.

Data logging. Implies data collection with storage for later data processing.

Hardware. The physical components of a computer system.

Software. Computer programs, whether inside or outside a computer.
Firmware. Computer programs stored in a semipermanent form, usually semiconductor memory, and used repeatedly without modification. Firmware can be changed only by replacing or removing hardware.

Pre-processor. A device in a data-acquisition system that performs a significant amount of data reduction by extracting specific information from raw signal representations in advance of the main processing operation. A pre-processor may constitute the whole of a data-acquisition interface, in which case it must also perform the data-acquisition task (conversion of spectrometer signal to computer representation), or it may specialize solely in data treatment.

Hard wired. A pre-processor that is capable of performing only certain defined tasks and no others without major physical modification.

Pre-programmed. A pre-processor that incorporates specific but readily alterable instructions to perform a particular task.

Signal conditioning. The process of altering the relationship of a transducer (ion or neutral detector) output with respect to time or other parameters (frequency, voltage, or current).

Signal processing. The mechanisms involved in analyzing, routing, sampling, or changing the representation of a signal.

Operational amplifier. A linear, high-gain dc voltage or current amplifier with high input impedance, low output impedance, and the capability of producing a bipolar output from a bipolar input.

Amplifier complex. A number of operational amplifiers configured for a specific function, packaged and used as a single unit.

Amplifier bandwidth. The range of signal frequencies over which an amplifier is capable of undistorted or unattenuated transmission. An operational amplifier should transmit de voltage accurately; the upper (bandwidth) limit is defined as the 3-db point (factor of 2 attenuation). Because bandwidth can vary with gain, the product of gain $x$ bandwidth can be a more useful parameter.

Amplifier noise. Can be of two kinds: white noise results from random fluctuations of signal over a power spectrum that contains all frequencies equally over a specified bandwidth; pink noise results when the frequencies diminish in a specified fashion over a specified range.

Differential amplifier. An operational amplifier with two inputs of opposite-gain polarity with respect to its 
output. Differential-output amplifiers with two opposite-sense outputs also exist.

Single-ended amplifier. An operational amplifier with a single input (or output).

Analog signal. A signal that can be expressed as a continuously variable mathematical function of time.

Digital signal. A signal that represents information in a computer-compatible form as a sequence of (binary) numbers that may describe discrete samples of an analog signal.

\section{Sample Introduction}

Sample introduction. The transfer of material to be analyzed into the ion source of a mass spectrometer before and/or during analysis.

Sample introduction system. A system used to introduce sample to a mass spectrometer ion source. "Sample introduction system," "introduction system," "sample inlet system," "inlet system," and "inlet" are synonymous terms.

Batch inlet. The historic term for a reservoir inlet. "Reservoir inlet" is preferred because a direct inlet probe is also a form of batch inlet. "Batch gas inlet" or "batch vapor inlet" are, however, completely descriptive terms.

Dual viscous-flow reservoir inlet. An inlet having two reservoirs, used alternately, each having a leak that provides viscous flow. This inlet is used for making precise comparisons of isotope ratios in two samples.

Continuous inlet. An inlet in which sample passes continuously into the mass spectrometer ion source, as distinguished from a reservoir inlet or a direct inlet probe.

Non-fractionating continuous inlet. An inlet in which gas flows from a gas stream being analyzed to the mass spectrometer ion source without any change in the conditions of flow through the inlet or by the conditions of flow through the ion source. This is usually viscous flow, such that the mean free path is very small in comparison with the smallest dimension of a traverse section of the channel. The flow characteristics are determined mainly by collisions between gas molecules, i.e., the viscosity of the gas. The flow may be laminar or turbulent.

Direct-inlet probe. A shaft or tube having sample holder at one end, which is inserted into the vacuum system of a mass spectrometer through a vacuum lock, to place the sample near to, at the entrance of, or within the ion source. The sample is vaporized by heat from the ion source, by heat applied from an external source, or by exposure to ion and/or atom bombardment. " Direct inlet probe," “'direct-introduction probe," or "direct-insertion probe" are synonymous terms. The use of DIP as an abbreviation for these terms is not recommended.

Vacuum-lock inlet. An inlet through which a sample is first placed in a chamber; the chamber is then pumped out and a valve is opened so that the sample can be introduced to the mass spectrometer ion source. A vacuum-lock inlet commonly uses a direct-inlet probe, which passes through one or more sliding seals, but other kinds of vacuum-lock inlets are used.

Direct-exposure probe. Provides for insertion of a sample on an exposed surface, such as a flat surface or a wire, into (rather than up to the entrance of) the ion source of a mass spectrometer.

Crucible direct-inlet prabe. Holds the sample in a cup-shaped device (the crucible) rather than on an exposed surface. A direct-inlet probe is assumed to be a crucible type unless otherwise specified.

GC/MS interface. An interface between a gas chromatograph and a mass spectrometer that provides continuous introduction of effluent gas from a gas chromatograph to a mass spectrometer ion source.

Direct GC/MS interface. An interface in which all the effluent from a gas chromatograph passes into the mass spectrometer ion source during an analysis, without any splitting of the effluent.

Splitter GC/MS interface. An interface in which the effluent from the gas chromatograph is divided before admission to the mass spectrometer, without enrichment of sample with respect to carrier gas.

Separator GC/MS interface. An interface in which the effluent from the gas chromatograph is enriched in the ratio of sample to carrier gas. "Separator," "molecular separator," and "enricher" are synonymous terms. A separator should generally be defined as an effusion separator, a jet separator, or a membrane separator.

Effusion separator (or effusion enricher). An interface in which carrier gas is preferentially removed from the gas entering the mass spectrometer by effusive flow (e.g., through a porous tube or through a slit). This is usually molecular flow, such that the mean free path is much greater than the largest dimension of a traverse section of the channel. The flow characteristics are determined by collisions of the gas molecules with surfaces; flow effects from molecular collisions are insignificant. 
Jet separator. An interface in which carrier gas is preferentially removed by diffusion out of a gas jet flowing from a nozzle. "Jet separator," "jet-orifice separator," "jet enricher," and "jet-orifice" are synonymous terms.

Membrane separator. A separator that passes gas or vapor to the mass spectrometer through a semi-permeable (e.g., silicon) membrane that selectively transmits organic compounds in preference to carrier gas. "Membrane separator," "membrane enricher," "semi-permeable membrane separator," and "semipermeable membrane enricher" are synonymous terms.

Solvent-divert system. Used in conjunction with an interface, it permits temporary interruption of the flow from a chromatograph to a mass spectrometer by briefly opening a valve to a pumping line. Thus, effluent present at a high concentration (usually solvent) does not enter the mass spectrometer ion source.

Liquid chromatograph / mass spectrometer (LC/MS) interface. An interface between a liquid chromatograph and a mass spectrometer that provides continuous introduction of the effluent from a liquid chromatograph to a mass spectrometer ion source.

Moving belt (ribbon or wire) interface. An interface that continuously applies all, or a part of, the effluent from a liquid chromatograph to a belt (ribbon or wire), which passes through two ur more orifices, with differential pumping, into the mass spectrometer's vacuum system. Heat is applied to remove the solvent and to evaporate the solute into the ion source.

Direct liquid introduction interface. An interface that continuously passes all, or a part of, the effluent from a liquid chromatograph to the mass spectrometer; the solvent usually functions as a chemical ionization agent for ionization of the solute.

Thermospray interface. Provides liquid chromatographic effluent continuously through a heated capillary vaporizer tube to the mass spectrometer. Solvent molecules evaporate away from the partially vaporized liquid, and analyte jons are transmitted to the mass spectrometer's ion optics. The ionization technique must be specified, e.g., pre-existing ions, salt buffer, filament, or electrical discharge.

Thermal desorption. The vaporization of ionic or neutral species from the condensed state by the input of thermal energy into the condensed state. The energy input mechanism must be specified.

Dynamic headspace GC/MS. The distillation of volatile and semivolatile compounds into a continuously flowing stream of carrier gas and into a device for trapping sample components. Contents of the trap are then introduced onto a gas chromatographic column. This is followed by mass spectrometric analysis of compounds eluting from the gas chromatograph.

Static headspace GC/MS. The partitioning of volatile and semivolatile compounds between two phases in a sealed container. An aliquot of the headspace gas generated is injected onto a gas chromatographic column. This is followed by mass spectrometric analysis of compounds eluting from the gas chromatograph.

\section{Scanning of Spectra}

Scanning method. The sequence of control over operating parameters of a mass spectrometer that results in a spectrum of masses, velocities, momenta, or energies.

Mass spectrum. A spectrum obtained when ions (usually in a beam) are separated according to the mass-to-charge $(\mathrm{m} / \mathrm{z})$ ratios of the ionic species present. This plot is a graphical representation of $\mathrm{m} / \mathrm{z}$ versus measured abundance information.

Momentum spectrum. A spectrum obtained when a beam of ions is separated according to the momentum-to-charge ratios of the ionic species contained within it. A magnetic sector analyzer achieves separation of the various ionic species in this way. If the ion beam is homogeneous in translational energy, as is the case with sector instruments, separation according to the $m / z$ ratios is also achieved.

Selected ion monitoring (SIM). Describes the operation of a mass spectrometer in which the ion currents at one (or several) selected $\mathrm{m} / \mathrm{z}$ values are recorded, rather than the entire mass spectrum. The use of the terms "multiple ion detection (MID)," multiple ion (peak) monitoring (MPM), and "mass fragmentography" are not recommended.

Ion kinetic energy spectrum. A spectrum obtained when a beam of ions is separated according to the translational energy-to-charge ratios of the ionic species contained within it. A radial electric field achieves separation of the various ionic species in this way.

Magnetic field scan. The usual method of producing a momentum (mass) spectrum in instruments.

Accelerating voltage (high voltage) scan. An alternative method of producing a momentum (mass) spectrum in magnetic deflection instruments. This scan can also be used, in conjunction with a fixed radial electric field, to produce an ion kinetic energy spectrum. 
Linked scan. A scan, in an instrument with two or more analyzers, in which two or more of the analyzer fields are scanned simultaneously so as to preserve a predetermined relationship between parameters that characterize these fields. Often these parameters are the field strengths, but may be the frequencies in the case of analyzers in which alternating fields are employed.

Linked scan at constant B/E. A linked scan at constant $B / E$ may be performed on a sector instrument that incorporates at least one magnetic sector plus one electric sector. It involves scanning the magnetic sector field-strength (B) and the electric sector fieldstrength (E) simultaneously, holding the accelerating voltage (V) constant, so as to maintain the ratio of the two fields strengths which transmit main-beam ions of predetermined mass-to-charge ratio. These preselected main-beam ions are the precursor (parent) ions whose fragment-ion spectrum is required. The fragmentation reactions observed occur in a field-free region traversed before the two sectors scanned in this way. This term " $\mathrm{B} / \mathrm{E}$ linked scan" is not recommended; it may suggest that the ratio $B / E$ varies during the scan.

Linked scan at constant $E^{2} / V$. A linked scan at constant $\mathrm{E}^{2} / \mathrm{V}$ may be performed on a sector instrument that incorporates at least one electric sector plus one magnetic sector. The electric sector field (E) and the accelerating voltage (V) are scanned simultaneously, so as to maintain the ratio $\mathrm{E}^{2} / \mathrm{V}$ at a constant value, equal to the value of this ratio which transmits the main beam of ions through the electric sector. The magnetic sector field is set at a fixed value such that main-beam ions of a predetermined $m / z$ are transmitted by the magnet; these preselected main-fragmentation reactions observed occur in a field-free region traversed before the two sectors scanned in this way. This term should not be used without prior explanation of the meaning of $E$ and $V$. The term " $E^{2} / V$ linked scan" is not recommended.

Linked scan at constant $B^{2} / E$. A linked scan at constant $B^{2} / E$ may be performed on a sector instrument that incorporates at least one electric sector plus one magnetic sector. It involves holding the accelerating voltage fixed and scanning the magnetic field (B) and the electric field (E) simultaneously so as to maintain the ratio $B^{2} / E$ at a constant value. This constant value corresponds to the ratio of the two fields which transmit main-beam ions of predetermined mass-tocharge ratio; these preselected main-beam ions are the fragment ions whose precursor ion spectrum is required. The fragmentation reactions thus observed occur in a field-free region traversed before the two sectors scanned in this way. This term should not be used without prior explanation of the meanings of $B$ and $\mathbf{E}$. The term " $B$ $/ E$ linked scan" is not recommended.

Linked scan at constant $B\left[1-\left(E / E_{o}\right)\right]^{1 / 2} / E$. A linked scan at constant $B\left[1-\left(E / E_{0}\right)\right]^{1 / 2} / E$ may be perfurmed on a sector instrument that incorporates at least one electric sector plus one magnetic sector. It involves holding the accelerating voltage fixed and scanning the magnetic field (B) and electric field (E) simultaneously, so as to maintain the quantity $B[1$ $\left.\left(\mathrm{E} / \mathrm{E}_{\mathrm{o}}\right)\right]^{1 / 2} / \mathrm{E}$ at a constant value. This constant value is equal to $B_{3} / E_{o}$, where $E_{0}$ and $B_{3}$ are, respectively, the electric sector field and magnetic sector field required to transmit $m_{3}$ ions in the main ion-beam; $m_{3}$ represents the mass $\left(m_{1}-m_{2}\right)$ of the selected neutral fragment whose precursor (parent) ion spectrum is required. The fragmentation reactions so observed occur in a field-free region traversed before the two sectors scanned in this way. This term should not be used without prior explanation of the meanings of $B$, $E$, and $E_{0}$. The term " $B\left[1-\left(E / E_{o}\right)\right]^{1 / 2} / E$ linked scan" is not recommended. The above three definitions are merely examples of the types of linked scan that might be used. Other linked scans can readily be defined in a similar manner.

\section{Fixed precursor ion scans (sector instruments).}

(a) Mass selection followed by ion kinetic energy analysis - If a precursor (parent) ion is selected by a magnetic sector all product (daughter) ions formed from it in the field-free region between the magnetic sector and a flowing electric sector can be identified by scanning an ion kinetic energy spectrum.

(b) Linked scan at constant $\mathrm{B} / \mathrm{E}$ or at constant $\mathrm{E}^{2} / \mathrm{V}-$ Both of these linked scans give a spectrum of all product (daughter) ions formed from a preselected precursor (parent) ion.

Fixed product ion scans (sector instruments). (a) high voltage scan, or (b) linked scan at constant $\mathbf{B}^{2} / \mathbf{E}-$ Both (a) and (b) give a spectrum of all precursor (parent) ions that fragment to yield a preselected product (daughter) ion.

Constant neutral loss (or fixed neutral fragment) scans. The linked scan at constant $B\left[1-\left(E / E_{o}\right)\right]^{1 / 2} / E$ gives a spectrum of all product (daughter) ions that have been formed by loss of a preselected neutral fragment from any precursor (parent) ions.

2E mass spectrum. Processes of the partial chargetransfer type

$$
\mathrm{m}^{2+}+\mathrm{N} \rightarrow \mathrm{m}^{+}+\mathrm{N}^{+}
$$

that occur in a collision cell (containing a gas, N) located in a field-free region preceding a magnetic and electric sector combination placed in either order may 
be detected as follows: If the instrument slits are wide, and if the electric sector field $E$ is set to twice the value required to transmit the main iun-beam, the only ions to be transmitted will be those with a kinetic energy-to-charge ratio twice, or almost exactly twice, that of the main ion-beam. The product ions of the process shown fulfill this condition. If the magnetic field $B$ is scanned, a mass spectrum of such singly charged product ions, and thus of their doubly charged precursors, is obtained. Such a spectrum is called a $2 \mathrm{E}$ mass spectrum.

E/2 mass spectrum. Processes of the charge-stripping type

$$
\mathrm{m}^{+}+\mathrm{N} \rightarrow \mathrm{m}^{2+}+\mathrm{N}+\mathrm{e}^{-}
$$

that occur in a collision cell (containing a gas, N) located in a field-free region preceding a magnetic and electric sector combination placed in either order may be detected as follows: If the instrument slits are wide, and if the electric sector field $\mathrm{E}$ is set to half the value required to transmit the main ion-beam, the only ions to be transmitted will be those with a kinetic energy-to-charge ratio half that of the main ion-beam. The product (daughter) ions of the charge-stripping process fulfill this condition. If the magnetic field $B$ is scanned, a mass spectrum of such doubly charged product ions, and thus their singly charged precursors, is obtained. Such a spectrum is called an E/2 mass spectrum. Interference from product ions from processes of the type

$$
\mathrm{m}_{1}^{+}+\mathrm{N} \rightarrow \mathrm{m}_{2}^{+}+\mathrm{N}+\left(\mathrm{m}_{1}-\mathrm{m}_{2}\right)
$$

where $m_{2}=0.5 m_{1}$, can arise in $F / 2$ mass spectra

Charge-inversion mass spectrum. Charge-inversion processes of the type

or

$$
\mathrm{m}^{+}+\mathrm{N} \rightarrow \mathrm{m}^{-}+\mathrm{N}^{2+}
$$

$$
\mathrm{m}^{-}+\mathrm{N} \rightarrow \mathrm{m}^{+}+\mathrm{N}+2 \mathrm{e}^{-}
$$

that occur in a collision cell (containing a gas, N) located in a field-free region preceding a magnetic and electric sector combination placed in either order may be detected as follows: If the instrument slits are wide, and if the connections to the two sectors, appropriate to transmission of either positive or negative main-beam ions, are simply reversed, the negative or positive product ions of the two processes, respectively, will be transmitted. If the magnetic field is scanned, a spectrum of such product ions will be obtained, and this spectrum is called a charge-inversion mass spectrum. These spectra are sometimes referred to as charge reversal, or as $-\mathrm{E}$ and $+\mathrm{E}$ spectra, respectively. The terms " $2 \mathrm{E}, \mathrm{E} / 2,-\mathrm{E}$, or $+\mathrm{E}$ mass spectrum" should not be used without prior explanation of the meaning of $2 E, E,+E$, and $-E$.

\section{Ion Detection and Sensitivity}

Detection of ions. The observation of electrical signals due to particular ionic species by a detector under conditions that minimize ambiguities from interferences. Ions may be detected by photographic or suitable electrical means.

Faraday cup (or cylinder) collector. A hollow collector, open at one end and closed at the other, used to measure the ion current associated with an ion-beam.

Electron multiplier. A device to multiply current in an electron beam (or in a photon or particle beam after conversion to electrons) by incidence of accelerated electrons upon the surface of an electrode. This collision yields a number of secondary electrons greater than the number of incident electrons. These electrons are then accelerated to another electrode (or another part of the same electrode), which in turn emits secondary electrons, continuing the process.

Photographic plate recording. The recording of ion currents (usually associated with ion-beams that have been spatially separated by $m / z$ values) by allowing them to strike a photographic plate, which is subsequently developed.

Detection limit. The detection limit of an instrument should be differentiated from sensitivity. The detection limit reflects the smallest flow of sample or the lowest partial pressure that gives a signal that can be distinguished from the background noise. One must specify the experimental conditions used and give the value of signal-to-noise ratio corresponding to the detection limit.

Base peak. The peak in a mass spectrum corresponding to the $m / z$ value that has the greatest intensity. This term may be applied to the spectra of pure substance or mixtures.

Intensity relative to base peak. The ratio of intensity of a particular peak in a mass spectrum to the intensity of the mass peak of the greatest intensity. This ratio is generally equated to the normalized ratio of the heights of the respective peaks in the mass spectrum, with the height of the base peak being taken as 100 .

Resolution: $70 \%$ valley definition, $m / \Delta m$. Let two peaks of equal height in a mass spectrum at masses $m$ and $m-\Delta m$ be separated by a valley that at its lowest point is just $10 \%$ of the height of either peak. For similar peaks at a mass exceeding $m$, let the height of the valley at its lowest point be more (by any amount) than $10 \%$ of either peak height. Then the resolution (10\% valley definition) is $\mathrm{m} / \Delta \mathrm{m}$. It is usually a function of $\mathrm{m}$, therefore $\mathrm{m} / \Delta \mathrm{m}$ should be given for $\mathrm{a}$ number of values of $\mathrm{m}$. 
Resolution: peak width definition, $m / \Delta m$. For a single peak made up of singly charged ions at mass $m$ in a mass spectrum, the resolution may be expressed as $\mathrm{m} / \Delta \mathrm{m}$, where $\Delta \mathrm{m}$ is the width of the peak at a height that is a specified fraction of the maximum peak height. It is recommended that one of three values $50 \%, 5 \%$, or $0.5 \%$ be used. For an isolated symmetrical peak, recorded with a system that is linear in the range between $5 \%$ and $10 \%$ levels of the peak, the $5 \%$ peak width definition is technically equivalent to the $10 \%$ valley definition. A common standard is the definition of resolution based upon $\Delta \mathrm{m}$ being full width of the peak at half its maximum height, sometimes abbreviated "FWHM."

Resolution energy. A value derived from a peak showing the number of ions as a function of their translational energy.

Resolving power (mass). The ability to distinguish between ions differing slightly in mass-to-charge ratio. It may be characterized by giving the peak width, measured in mass units, expressed as a function of mass, for at least two points on the peak, specifically for $50 \%$ and for $5 \%$ of the maximum peak height.

\section{Total ion current (TIC).}

(a) after mass analysis-the sum of all the separate ion currents carried by the different ions contributing to the spectrum.

(b) before mass analysis-the sum of all the separate ion currents for ions of the same sign.

Sensitivity. Different measures of sensitivity are recommended, depending on the nature of the sample, and the required inlet system. The first, which is suitable for relatively involatile materials as well as gases, depends upon the observed change in ion current for a particular change of flow rate of sample through the ion source. The recommended unit is coulomb per microgram. A second method of stating sensitivity, most suitable for gases, depends upon the change of ion current relative to the change of partial pressure of the sample in the ion source. The recommended unit is ampere Pascal ${ }^{-1}$. It is important that the relevant experimental conditions corresponding to sensitivity measurement should always be stated. These typically include details of the bombarding electron current, slit dimensions, angular collimation, gain of the detector, scan speed, mass range scanned, and whether the measured signal corresponds to a single mass peak or to the ion-beam integrated over a specified mass range. Sample flow into the ion source per unit time should be noted; indication of the time involved in the determination should also be given, i.e., counting time or bandwidth. The sensitivity should be differentiated from the detection limit.

\section{Ionization Nomenclature}

Electron ionization. Ionization of any species by electrons. The process may be written:

$$
\mathrm{M}+\mathrm{e}^{-} \rightarrow \mathrm{M}^{+\cdot}+2 \mathrm{e}^{-} \text {for atoms or molecules, }
$$

and

$$
M^{+}+\mathbf{e}^{-} \rightarrow \mathrm{M}^{+}+2 \mathrm{e}^{-} \text {for radicals. }
$$

Photo-ionization. Ionization of any species by photons:

$$
\mathrm{M}+\mathrm{hv} \rightarrow \mathrm{M}^{+\cdot}+\mathrm{e}^{-}
$$

Electrons and photons do not "impact" molecules or atoms. They interact with them in ways that result in various electronic excitations including ionization. For this reason it is recommended that the terms "electron impact" and "photon impact" be avoided.

Field ionization. The removal of electrons from any species by interaction with a high electrical field.

Field desorption. The formation of ions in the gas phase from a material deposited on a solid surface (known as an emitter) that is placed in a high electrical field. "Field desorption" is an ambiguous term because it implies that the electric field desorbs a material as an ion from some kind of emitter on which the material is deposited. There is growing evidence that some of the ions formed are due to thermal ionization, and some to field ionization of material vaporized from the emitter. Because there is little or no ionization unless the emitter is heated by an electric current, "field desorption" is a misnomer. However, the term is furmly implanted in the literature and most users (by no means all) understand what is meant regardless of the implications of the term. Because no better simple term has been suggested to take its place, it is recommended with reluctance that it be retained.

\section{Chemi-ionization. A process:}

$$
\mathrm{A}^{*}+\mathrm{M} \rightarrow \mathrm{AM}^{+}+\mathrm{e}^{-}
$$

whereby gaseous molecules are ionized when they interact with other internally excited gaseous molecules or molecular moieties. Chemi-ionization and chemical ionization must not be used interchangeably.

Chemical ionization. The formation of new ionized species when gaseous molecules interact with ions. The process may involve transfer of an electron, a proton, or other charged species betwreen the reactants. When a positive ion results from chemical ionization (CI), the term may be used without qualification. When a negative ion results, the term "negative ion chemical ionization" can be substituted. Specifics 
relating to the ionization should be given, e.g., if negative ions are formed from sample molecules via resonance capture of thermal electrons generated in a CI source, this should be specified.

Surface ionization. Takes place when an atom or molecule is ionized when it interacts with a solid surface. Ionization occurs only when the work function of the surface, the temperature of the surface, and the ionization energy of the atom or molecule have an appropriate relationship.

Thermal ionization. Takes place when an atom or molecule interacts with a heated surface or is in a gaseous environment at high temperatures. Examples of the latter may be a capillary arc plasma, a microwave plasma, or an inductively coupled plasma.

Atmospheric pressure ionization. Chemical ionization performed at atmospheric pressure.

Spark (source) ionization. Occurs when a solid sample is vaporized and partially ionized by an intermittent electric discharge. Further ionization occurs in the discharge when gaseous atoms and small molecular moieties interact with energetic electrons in the intermittent discharge. It is recommended that the word "source" be dropped from this term.

Auto ionization. Occurs when an internally supraexcited atom or molecular mojety loses an electron spontaneously without further interaction with an energy source. (The state of the atom or molecular moiety is known as a pre-ionization state.)

Associative ionization. Occurs when two excited gaseous atoms or molecular moieties interact and the sum of their internal energies is sufficient to produce a single, additive ionic product.

Multi-photon ionization. Occurs when an atom or molecule and its associated ions have energy states in which they can absorb the energy in two or more photons.

Penning ionization. Occurs through the interaction of two or more neutral gaseous species, at least one of which is internally excited:

$$
\mathrm{A}^{*}+\mathrm{M} \rightarrow \mathrm{A}+\mathrm{M}^{+}+\mathrm{e}^{-}
$$

Charge-exchange (charge transfer) ionization. Occurs when an ion/atom or ion/molecule reaction takes place in which the charge on the ion is transferred to the neutral species without any dissociation of either.

Dissociative charge transfer. Occurs when an jon/molecule reaction takes place in which the charge on the ion is transferred to the neutral species. The new ion then dissociates to one or more fragment ions.

Ion-pair formation. An ionization process in which a positive fragment ion and a negative fragment ion are the only products.

Ionization cross section. A measure of the probability that a given ionization process will occur when an atom or molecule interacts with an electron or a photon.

Electron attachment. A resonance process whereby an electron is incorporated into an atomic or molecular orbital of an atom or molecule.

Ionization energy. The minimum energy of excitation of an atom, a molecule, or a molecular moiety that is required to remove an electron in order to produce a positive ion.

Vertical ionization. A process whereby an electron is removed from a molecule, in its ground state or an excited state, so rapidly that a positive ion is produced without change in the positions or momenta of the atoms. The resultant ion is often in an excited state.

Adiabatic ionization. A process whereby an electron is removed from the ground state of an atom or molecule, producing an ion in its ground state.

Ionization. A process that produces an ion from a neutral atom or molecule.

Dissociatioe ionization. A process in which a gaseous molecule decomposes to form products, one of which is an ion.

Ionic dissociation. Decomposition of an ion into another ion of lower formula weight, plus one or more neutral species.

Ionization efficiency. The ratio of the number of ions formed to the number of electrons, photons, or particles that are used to produce ionization.

Ionization efficiency curve. Shows the number of ions produced as a function of energy of the electrons, photons, or particles used to produce ionization.

Laser ionization. Occurs when a sample is irradiated with a laser beam. In the irradiation of gaseous samples, ionization occurs via a single- or multi-photon process. In the case of solid samples, ionization occurs via a thermal process.

Desorption ionization (DI). General term to encompass the various procedures (e.g., secondary ion mass 
spectrometry, fast atom bombardment, californium fission fragment desorption, thermal desorption) in which ions are generated directly from a solid or liquid sample by energy input. Experimental conditions must be clearly stated.

Appearance energy. The minimum energy that must be imparted to an atom, molecule, or molecular moiety in order to produce a specified ion. The use of the alternative term "appearance potential" is not recommended.

Electron energy. The potential difference through which electrons are accelerated before they are used to bring about electron ionization. The term "ionizing voltage" is sometimes used in place of "electron energy."

\section{Types of Ions, Ion Structures}

Positive ion. An atom, radical, molecule, or molecular moiety that has lost one or more electrons, acquiring an electrically positive charge. The use of the term "cation" as an alternative is not recommended, except in the context of the chemical reactions or structures. The use of "mass ion" is not recommended.

Negative ion. An atom, radical, molecule, or molecular moiety that has gained one or more electrons, acquiring an electrically negative charge. The use of the term "anion" as an alternative is not recommended, except in the context of chemical reactions or structures.

Singly, doubly, triply, etc. charged ion. An atom, molecule, or molecular moiety that has gained or lost one, two, three, or more electrons. The term multiply charged ion is used to refer to ions that have gained or lost more than one electron where the number of electrons lost or gained is not designated.

Parent ion. An electrically charged molecular moiety that may dissociate to form fragments, of which one or more may be electrically charged, and one or more are neutral species. A parent ion may be a molecular ion or an electrically charged fragment of a molecular ion.

Daughter ion. An electrically charged product of reaction of a particular parent ion. In general, such ions have a direct relationship to a particular precursor ion and indeed may relate to a unique state of the precursor ion. The reaction need not necessarily involve fragmentation. It could, for example, involve a change in the number of charges carried. Thus, all fragment ions are daughter ions but not all daughter ions are necessarily fragment ions.
Fragment ion. An electrically charged dissociation product of an ionic fragmentation. Such an ion may dissociate further to form other electrically charged molecular or atomic moieties of successively lower formula weight.

Rearrangement ion. An electrically charged dissociation product, of a molecular or parent ion, in which atoms or groups of atoms have transferred from one portion of the molecule or molecular moiety to another during the fragmentation process.

Stable ion. An ion that is not sufficiently excited to dissociate into a daughter ion and associated neutral fragment(s), or to react further in the time frame of the mass spectrometric analysis under stated experimental conditions.

Unstable ion. An ion that is sufficiently excited to dissociate within the ion source, under stated experimental conditions.

Metastable ion. An ion that is sufficiently excited to dissociate into a particular daughter ion and neutral species during the flight from the ion source to the detector. The dissociation is most readily observed when it takes place in one of the field-free regions in a mass spectrometer.

\section{Precursor ion. Synonymous with parent ion.}

Product ion. Synonymous with daughter ion.

Progeny fragment ions. Includes daughters, granddaughters, great-granddaughters, etc. It is a more generic term than daughter or product ion. Given the sequential fragmentation scheme:

$$
\begin{aligned}
\mathrm{M}_{1}^{+} & \rightarrow \mathrm{M}_{2}^{+} \rightarrow \mathrm{M}_{3}^{+} \rightarrow \mathrm{M}_{5}^{+} \\
\mathrm{M}_{4}^{+} & =\text {parent to } \mathrm{M}_{5}^{+} \\
& =\text {daughter and granddaughter of } \mathrm{M}_{2}^{+} \\
& =\text {daughter and great-granddaughter of } \mathrm{M}_{1}^{+} \\
\mathrm{M}_{3}^{+} & =\text {parent to } \mathrm{M}_{4}^{+} \\
& =\text {daughter and granddaughter of } \mathrm{M}_{1}^{+} \\
& =\text {daughter of } \mathrm{M}_{2}^{+}
\end{aligned}
$$

Principal ion. A molecular or fragment ion that is made up of the most abundant isotopes of each of its atomic constituents. In the case of compounds that have been artificially isotopically enriched in one or more positions (such as $\mathrm{CH}_{3}{ }^{13} \mathrm{CH}_{3}$, or $\mathrm{CH}_{2} \mathrm{D}_{2}$ ), the principal ion may be defined by treating the heavy 
isotopes as new atomic species. Thus, in the above two examples, the principal ions would be of masses 31 and 18 , respectively.

Molecular ion. An ion formed by the removal (positive ions) or addition (negative ions) of one or more electrons from a molecule without fragmentation of the molecular structure. The mass of this ion corresponds to the sum of the masses of the most abundant naturally occurring isotopes of the various atoms that make up the molecule (with a correction for the masses of the electrons lost or gained). For example, the mass of the molecular ion of the ethyl bromide, $\mathrm{C}_{2} \mathrm{H}_{5} \mathrm{Br}$ will be $2 \times 12$ plus $5 \times 1.0078246$ plus 78.91839 minus the mass of the electron $\left(\mathrm{m}_{e}\right)$. This is equal to $107.95751 \mu-\mathrm{m}_{\mathrm{e}}, \mu$ being the unit of atomic mass based on the standard that the mass of the isotope ${ }^{12} \mathrm{C}=12 \mu$ exactly.

Isotopic molecular ion. A molecular ion containing one or more of the less abundant naturally occurring isotopes of the atoms that make up the molecular strurture. Thus, for ethyl bromide there exist molecular isotope ions such as ${ }^{13} \mathrm{CCH}_{5} \mathrm{Br}^{+} ; \mathrm{C}_{2} \mathrm{H}_{4} \mathrm{DBr}^{+}$; $\mathrm{C}_{2} \mathrm{H}_{5}{ }^{81} \mathrm{Br}^{+*}$; ${ }^{13} \mathrm{C}_{2} \mathrm{H}_{5}{ }^{81} \mathrm{Br}^{+*}$ etc.

Isotopic ion. Any ion containing one or more of the less abundant naturally occurring isotopes of the elements that make up its structure.

Isotopically enriched ion. Any ion containing an abundance of a particular isotope above the level at which it occurs in nature.

Dimeric ion. An ion formed either when a chemical species exists in the vapor phase as a dimer and can be detected as such, or when a molecular ion can attach to a neutral molecule within the ion source to form an ion such as $\left[2 \mathrm{M}^{+*}\right.$, where $M$ represents the molecule.

Protonated molecule. An ion formed by interaction of a molecule with a proton abstracted from an ion, as often occurs in chemical ionization according to the reaction:

$$
\mathrm{M}+\mathrm{XH}^{+} \rightarrow \mathrm{MH}^{+}+\mathrm{X}
$$

The symbolism $[\mathrm{M}+\mathrm{H}]^{+}$may also be used to represent the protonated molecule. The widely used term "protonated molecular ion" to describe the $\mathrm{MH}^{+}$ion is not recommended because it suggests an association product of a proton with a molecular ion.

Adduct ion. An ion formed by interaction of two species, usually an ion and a molecule, and often within the ion source, to form an ion containing all the constituent atoms of one species as well as an additional atom or atoms.
Cluster ion. An ion formed by the combination of two or more molecules of a chemical species, often in association with a second species. For example, $\left[\left(\mathrm{H}_{2} \mathrm{O}\right)_{\mathbf{n}} \mathrm{H}\right]^{+}$is a cluster ion.

Radical ion. An ion containing an unpaired electron that is thus both an ion and a free radical. The presence of the odd electron is denoted by placing a dot alongside the symbol for the charge. Thus, $\mathrm{C}_{2} \mathrm{H}_{6}^{+}$. and $\mathrm{SF}_{6}^{-}$are radical ions.

\section{Odd-electron ion. Synonymous with radical ion.}

Evenelectron ion. An ion containing no unpaired electrons, e.g., $\mathrm{CH}_{3}{ }^{+}$in its ground state.

Ion structures. Capital letters enclosed in parentheses should be used to represent an ion that contains different structural features; the charge should be located outside the parentheses. Thus, one should write $(\mathrm{ABC})^{+}$to refer to an even-electron species, or $(\mathrm{ABC})^{+\cdot}$ to emphasize that one is dealing with an odd-electron species. An exception is that an ion may be written, for example, $\left(A^{+} B C\right)$ or $\left(A^{+} B C\right)$ if it is necessary to discuss structures in which the charge and the odd electron are localized. The empirical formula of a molecular ion may be written in four ways: The molecular ion of aniline, for example, may be written $\left(\mathrm{C}_{6} \mathrm{H}_{7} \mathrm{~N}\right)^{+\cdot}$ or it may be written $\mathrm{C}_{6} \mathrm{H}_{5} \mathrm{NH}_{2} 7^{+-}$to emphasize that one is discussing an ion formed from a molecule with the structure $\mathrm{C}_{6} \mathrm{H}_{5} \mathrm{NH}_{2}$ by the removal of a single electron, the upper half of a bracket being used to indicate that the structure of the ion may or may not be the same as that of the molecule from which it was formed. If one wishes to discuss a particular structure in which an odd electron is localized, then one may write $\left(\mathrm{C}_{6} \mathrm{H}_{5} \mathrm{~N}^{+} \mathrm{H}_{2}\right)$. The electron ionization process of aniline, for example, may be written:

$$
\begin{aligned}
\mathrm{C}_{6} \mathrm{H}_{5} \mathrm{HN}_{2}+\mathrm{e}^{-} & \left.\rightarrow \mathrm{C}_{6} \mathrm{H}_{5} \mathrm{NH}_{2}\right\rceil^{+\cdot}+2 \mathrm{e}^{-} \\
& \rightarrow\left(\mathrm{C}_{6} \mathrm{H}_{7} \mathrm{~N}\right)^{+-}+2 \mathrm{e}^{-}
\end{aligned}
$$

Structural formulae of ions in which it is desired to indicate the localization of an odd electron may, alternatively, be written without the parentheses. Thus,

$$
\mathrm{C}_{6} \mathrm{H}_{5}+\mathrm{N} \cdot \mathrm{H}_{2} \text { or: }
$$

Bond fission. Confusion can arise when a hyphen is used in the symbolism. Thus, $D(R-X)$ has been used to mean the dissociation energy of the bond between $\mathrm{R}$ and $\mathrm{X}$ while $\left(\mathrm{X}-\mathrm{CH}_{2}\right)^{+}$might mean the 
next higher homolog of $\mathrm{X}^{+}$or the ion formed from $\mathrm{X}^{+}$ by removal of a $\mathrm{CH}_{2}$ group. Thus, it is recommended that a dash should not be used to indicate a bond, except in a conventional structural formula such as that for the acetone molecular ion shown above this paragraph. In other cases, the next higher homolog of $\mathrm{X}^{+}$shoud be written $\left(\mathrm{XCH}_{2}\right)^{+}$, without any dash. In the event that it is necessary to emphasize that a bond is breaking, it should be represented by two dots and a wavy line (no other bond than the breaking bond being illustrated):

$$
\left[\mathrm{R} ; \mathrm{S}_{1} \cdot \mathrm{CH}_{2} \mathrm{CH}_{2} \mathrm{R}_{2}\right]^{+\cdot}
$$

When, in addition to indicating fragmentation of the bond, it is necessary to emphasize the mass number of the fragments formed, this is done by writing the mass number at the top (right-hand fragment) or the bottom (left-hand fragment) as shown:

$$
\mathrm{CH}_{3} \mathrm{CH}_{29} \cdot S \cdot \mathrm{CH}_{2} \mathrm{CH}_{27} \cdot S^{47} \cdot \mathrm{CH}_{2} \mathrm{SH}^{+\cdot}
$$

Loss of a particular group should be indicated by the use of a minus sign located outside the parentheses or to the right of the "+"' sign. Spaces should be left on either side of the minus sign to reduce any confusion as to its meaning. Thus one would write

$$
(\mathrm{M})^{+\cdot}-\mathrm{CH}_{2} \text { or } \mathrm{C}_{6} \mathrm{H}_{5} \mathrm{CH}_{3} \mathrm{l}^{+\cdot}-\mathrm{H}^{\cdot}
$$

It is recommended that the convention used by Budzikiewicz, Djerassi, and Williams [Mass Spectrometry of Organic Compounds, Holden-Day Inc., 1967: p. 2] be followed in referring to $\alpha$-cleavage as: "fission of a bond originating at an atom which is adjacent to the one assumed to bear the charge; the definition of $\beta$-, $\gamma-$, then follows automatically." The process

$$
\stackrel{\mathrm{O}^{+\cdot}}{\|} \rightarrow \stackrel{+}{\mathrm{C}} \equiv \mathrm{C}-\mathrm{R}_{2}+\mathrm{R}_{1}
$$

would thus be described as " $\alpha$-fission of a ketone with explusion of a radical $R_{1} . "$ The carbon atoms of the radical $R_{1}$ are called the $\alpha-, \beta-, \gamma$ - carbons, starting with the atom nearest the functional group.

The symbol ' $\_$' is recommended for indicating the movement of two electrons (heterolysis).

The symbol ' $\longrightarrow$ ' is recommended for indicating the movement of one electron (homolysis).

Rearrangement reactions. May be skeletal, in which case the symbol $\longrightarrow$ is employed:

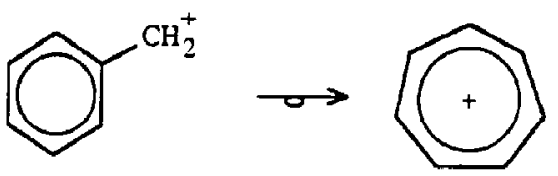

They may also involve the specific transfer of atom(s) within a molecule together with bond fission. An example of this is the McLafferty Rearrangement, which is defined as $\beta$-cleavage with concomitant specific transfer of a $\gamma$-hydrogen atom in a six-membered transition state in monounsaturated systems regardless of whether the rearrangement is formulated by a radical or by an ionic mechanism, and irrespective of which fragment retains the charge.

\section{Ion / molecule Reactions}

Ion/neutral reaction. Interaction of a charged species with a neutral reactant to produce either chemically different species or changes in the internal energy of one or both of the reactants.

Ion/molecule reaction. An ion/neutral reaction in which the neutral species is a molecule.

Charge-inversion reaction. An ion/neutral reaction wherein the charge on the reactant ion is reversed in sign.

Charge-transfer reaction. An ion/neutral reaction wherein the total charge on the reactant ion is transferred to the reactant neutral species so that the reactant ion becomes a neutral entity.

Partial charge-transfer reaction. An ion/neutral reaction that reduces the charge on a multiply charged reaction ion.

Charge-stripping reaction. An ion/neutral reaction that increases the positive charge on the reactant ion.

Charge-permutation reaction. An ion/neutral reaction involving a change in the magnitude and/or sign of the charges on the reactants. Some of the possible reactions of ions $\mathrm{M}^{2+}, \mathrm{M}^{+}$and $\mathrm{M}^{-}$with a neutral species $\mathrm{N}$ would be categorized on the basis of the above definitions as follows (all are ion/neutral reactions and also charge-permutation reactions):

$$
\begin{array}{ll}
\mathrm{M}^{2+}+\mathrm{N} \rightarrow \mathrm{M}^{+}+\mathrm{N}^{+} & \text {(partial charge-transfer) } \\
\mathrm{M}^{+}+\mathrm{N} \rightarrow \mathrm{M}^{2+}+\mathrm{N}+\mathrm{e}^{-} & \text {(charge-stripping) } \\
\mathrm{M}^{-}+\mathrm{N} \rightarrow \mathrm{M}^{+}+\mathrm{N}+2 \mathrm{e}^{-} & \begin{array}{c}
\text { (charge-stripping and } \\
\text { charge-inversion) }
\end{array}
\end{array}
$$

Collision-induced dissociation (or decomposition), abbreviated CID. An ion/neutral process wherein the (fast) 
projectile ion is dissociated as a result of interaction with a target neutral species. This is brought about by conversion during the collision of part of the translational energy of the ion to internal energy in the ion. The term collisional-activated dissociation (or decomposition), abbreviated CAD, is also used.

Collisional activation. An ion/neutral process wherein excitation of a (fast) projectile ion is brought about by the same mechanism as in collision-induced dissociation. (The ion may decompose subsequently.)

Collisional excitation. An ion/neutral process wherein the (slow) reactant ion's internal energy increases at the expense of the translational energy of either (or both) of the reacting species. The scattering angle may be large.

Elastic scattering. An ion/neutral interaction wherein the direction of motion of the ion is changed, but the total translational energy or internal energy of the collision partners remains the same.

Inelastic scattering. An ion/neutral interaction wherein the direction of motion of the ion is changed, and the total translational energy of the collision partners is reduced.

Elastic collision. A collision resulting in elastic scattering.

Inelastic collision. A collision resulting in inelastic scattering.

Superelastic collision. A collision that increases the translational energy of the fast-moving collision partner.

Ionizing collision. An ion/neutral reaction in which an electron or electrons are stripped from the ion and/or the neutral species in the collision. Generally, this term describes collisions of fast moving ions or atoms with a neutral species in which the neutral species is ionized. Care should be taken to emphasize if charge-stripping of the ion has taken place.
Assaciation reaction (associative combination). The reaction of a (slow moving) ion with a neutral species wherein the reactants combine to form a single ionized species.

Ion/neutral exchange reaction. An association reaction that subsequently or simultaneously liberates a different neutral species.

Translational spectroscopy. A technique to investigate the distribution of the velocities of product ions from ion/neutral reactions.

Ion energy loss spectrum. A spectrum that shows the loss of translation energy among ions involved in ion/neutral reactions.

Impact parameter. The distance between two particles at their closest approach, had they had continued in their original direction at their original speeds.

Interaction distance. The greatest distance between two particles at which it is discernible that they will not pass at the impact parameter.

Charge-exchange reaction. Synonymous with charge-transfer reaction.

Partial charge-exchange reaction. Synonymous with partial charge-transfer reaction.

\section{Vacuum}

The separate section of vacuum terms has been discontinued. The most recent listing of accepted terms from the American Vacuum Society is now a monograph that covers "vacuum science and technology, surface science, thin film technology, vacuum metallurgy, and electronic materials." It may be ordered for $\$ 10.00$ from AVS:

Dictionary of Terms for Areas of Science and Technology Seroed by the American Vacuum Society, 2d ed., 1984. ISBN 0-88318-456-7.

Marion Churchill

American Vacuum Society

335 East 45th Street

New York, NY 10017 